
AMORPHOUS SEMICONDUCTOR MODELS

M. M. J. Treacy, NEC Research Institute, Inc., 4 Independence Way, Princeton, NJ

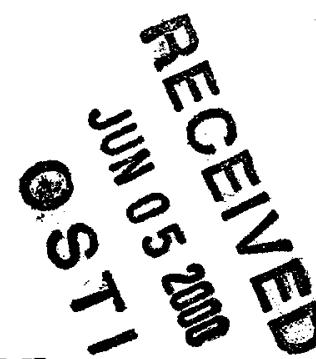

P. M. Voyles, Department of Physics, University of Illinois at UrbanaChampaign, 1110 West Green Street, Urbana, IL 61801

J. Murray Gibson, Materials Science Division, Argonne National Laboratory, 9700 South Cass Avenue, Argonne, IL 60439-4838

April, 2000

The submitted manuscript has
been created by the University of
Chicago as Operator of Argonne
National Laboratory ("Argonne")
under Contract No. W-31-109-
ENG-38 with the U.S. Department
of Energy. The U.S. Government
retains for itself, and others acting
on its behalf, a paid-up, non
exclusive, irrevocable worldwide
license in said article to reproduce,
prepare derivative works,
distribute copies to the public, and
perform publicly and display
publicly, by or on behalf of the
Government.

Distribution:

1-2. PRS

3. J. M. Gibson

4. L. E. Rehn

5. J. Coble

6. File

Conference Paper to be published in the proceedings of the Materials Research Society's Spring 2000 Meeting held April 24-28, 2000, in San Francisco, CA.

*Work supported by the U.S. Department of Energy, Office of Science under contract No. W-31-109-Eng-38. 


\section{DISCLAIMER}

This report was prepared as an account of work sponsored by an agency of the United States Government. Neither the United States Government nor any agency thereof, nor any of their employees, make any warranty, express or implied, or assumes any legal liability or responsibility for the accuracy, completeness, or usefulness of any information, apparatus, product, or process disclosed, or represents that its use would not infringe privately owned rights. Reference herein to any specific commercial product, process, or service by trade name, trademark, manufacturer, or otherwise does not necessarily constitute or imply its endorsement, recommendation, or favoring by the United States Government or any agency thereof. The views and opinions of authors expressed herein do not necessarily state or reflect those of the United States Government or any agency thereof. 


\section{DISCLAIMER}

Portions of this document may be illegible in electronic image products. Images are produced from the best available original document. 


\title{
Topological Signatures of Medium Range Order in Amorphous Semiconductor Models
}

\author{
M. M. J. Treacy*, P. M. Voyles* ${ }^{\dagger}$ and J. M. Gibson ${ }^{\#}$ \\ *NEC Research Institute, Inc. 4 Independence Way, Princeton, NJ 08540 \\ ${ }^{\dagger}$ Dept. of Physics, University of Illinois, 1110 W. Green St., Urbana, IL 61801 \\ "Materials Science Division, Argonne National Laboratory, 9700 Cass Ave., Argonne, IL 60439
}

\begin{abstract}
The topological local cluster (or Schläfli cluster) concept of Marians and Hobbs is used to detect topologically crystalline regions in models of disordered tetrahedral semiconductors. We present simple algorithms for detecting both Wells-type shortest circuits and O'Keeffe-type rings, which can be used to delineate alternative forms of the Schläfli cluster in models.
\end{abstract}

\section{INTRODUCTION}

Fluctuation microscopy experiments have shown that evaporated amorphous silicon and germanium exhibit a paracrystalline-type structure [1], in which some regions possess the bonding topology of the crystal, but are strained so that the component atoms do not lie on a lattice. This discovery was aided by comparison of the fluctuation microscopy data to simulations from molecular dynamics structures, and has highlighted the need for effective tools for characterizing medium range order in structural models. To address this need, useful topological analysis tools for characterizing models have been developed [2-6].

In this paper, we describe how the local cluster (or Schläfli cluster) concept of Marians and Hobbs [2] can be used to detect paracrystalline regions in models of disordered materials. We also outline a simple algorithm for detecting these topological clusters.

\section{TOPOLOGICAL REPRESENTATION OF NETWORK STRUCTURES}

Because of their periodicity, crystals can be completely defined by the positions of a small asymmetric unit of atoms and a group of symmetry operators. In principle, the bonding topology of covalent materials, such as perovskites and zeolites, can also be represented unambiguously by a graph. Atoms define the graph vertices and bonds define the graph edges. Such a topological approach has advantages for disordered models because it is insensitive to local strain and symmetry breaking.

One useful topological descriptor that is used extensively in zeolite studies is the circuit symbol [7-9], which is an ordered list of all the shortest closed paths that pass through each vertex and all the pairwise combinations of that vertex's first nearest neighbors. If there are $N$ nearest neighbors, there are $N(N-1) / 2$ distinct sets of circuits. The shortest meaningful circuit is of length 3. The circuit symbol itself does not give a unique description of any given topology. However, when used in conjunction with other topological descriptors such as the coordination sequence, there is a very high likelihood that graphs with the same descriptors represent the same topology. The coordination sequence is a list of the number of vertices $N_{p}$ in coordination shell $p$ around each atom [8-10]. However, coordination sequences are less useful for describing local topology in amorphous models because they are sensitive to long-range disorder. In zeolite studies, there are two definitions of a circuit that are commonly used. The Wells-type circuit [7] 
is the shortest circuit possible, whereas the O'Keeffe-type circuit [8] is the shortest ring or fundamental circuit. A fundamental circuit has the property that for any two vertices on the circuit there is no shorter path between them than the path that lies on the circuit [2]. For the central vertex and any pair of its neighbors, there may be more than one circuit that satisfies these criteria.

As pointed out by Marians and Hobbs [2], circuit symbols are potentially useful for characterizing amorphous materials. In particular, it is expected that the paracrystalline regions in amorphous $\mathrm{Si}$ and $\mathrm{Ge}$ will have predominantly cubic, or perhaps hexagonal, diamond bonding topology [1], each of which should have readily identifiable signatures. In zeolite studies, the circuit descriptor for each nearest-neighbor pair of atoms is represented in the Schläfli notation, $C_{n}$, where $C$ is the circuit size, and $n$ is the number of circuits of that length [8]. The circuit symbol is an ordered list of these circuit descriptors. Both the Wells and O'Keeffe forms of the Schläfli circuit symbol for the cubic diamond structure are given by $6_{2} \cdot 6_{2} \cdot 6_{2} \cdot 6_{2} \cdot 6_{2} \cdot 6_{2}$. This notation tells us that there are six distinct sets of circuits (since the number of bonded neighbors is $N=4$, and $N(N-1) / 2=6$ ) and each set contains two 6-circuits. Figure 1a depicts the atoms that lie on these circuits for the cubic diamond structure. The black circle, labeled 29, is the central vertex, and the four gray circles are the first nearest neighbors. For the pair 1 and 2 , there are two six-ring circuits that are given by the paths $29,1,10,11,12,2,29$ and 29, 1, 25, 27, 23, 2, 29. Both rings have the "chair" conformation and the pair is assigned the symbol $6_{2}$. The other five sets of circuits are equivalent. The hexagonal lonsdaleite structure (Figure $1 \mathrm{~b}$ ) has the same circuit symbol $6_{2} \cdot 6_{2} \cdot 6_{2} \cdot 6_{2} \cdot 6_{2} \cdot 6_{2}$. However, the two six-rings associated with vertex pairs 1 and 2 have the "chair" $(27,1,10,11,12,2,27)$ and "boat" $(27,1,25,24,23,2,27)$ conformations. Further, all three sets of $6_{2}$ circuits involving neighbor number 4 comprise two "boat" conformation 6-rings. Thus, unlike the cubic structure, the hexagonal structure has two inequivalent sets of circuits involving two types of ring.

Clearly, the circuit symbol alone is insufficient to distinguish the cubic and hexagonal topologies. For example, there is no information about the "boat" and "chair" conformations. For the unfaulted cubic and hexagonal frameworks, the coordination sequences are $4,12,24,42 \ldots$ and $4,12,25,44 \ldots$ respectively. The sequences do not resolve until the third shell, and about one half of the third-shell atoms are not part of the Schläfli cluster. The coordination sequence is clearly an unsuitable descriptor of local topology. To help circumvent such ambiguities, we proposed that the topology of the whole cluster of atoms encompassed by the Schläfli circuit
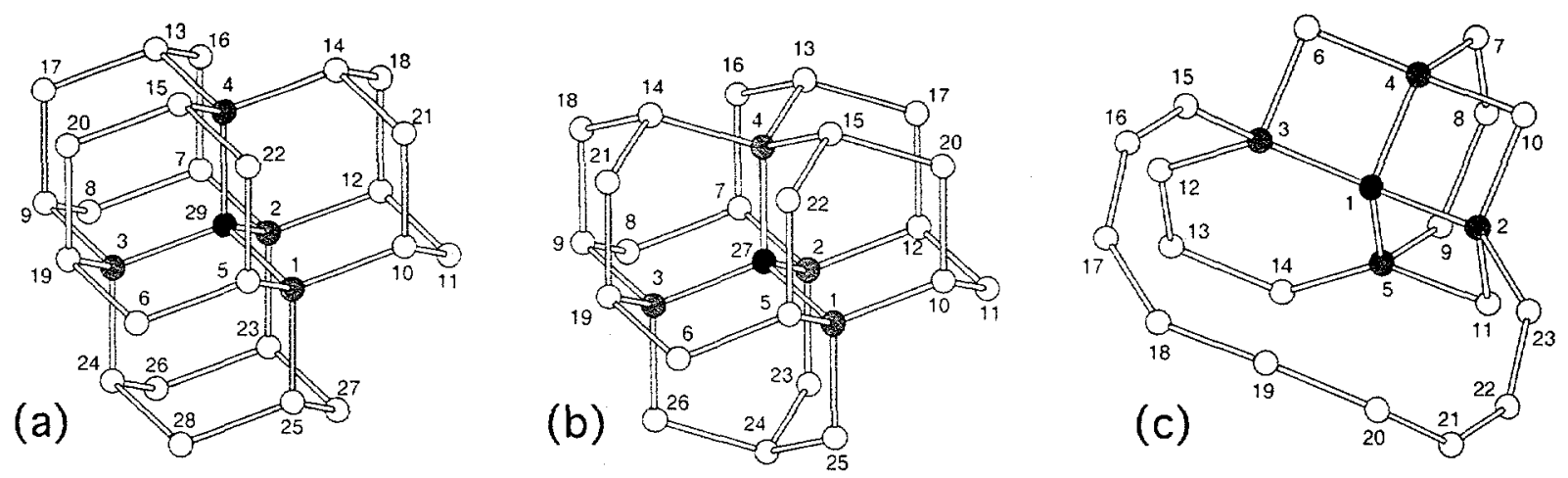

Figure 1. Example Schläfli clusters for(a) the cubic diamond framework, (b) the hexagonal diamond (lonsdaleite) framework, (c) faujasite (FAU). The O'Keeffe form of the Schläfli cluster, of weight 23, is shown. The Wells form, of weight 14, excludes vertices 15-23. 
symbol be considered [6]. We called this cluster the Schläfli cluster and also proposed that it is the smallest topologically meaningful crystalline unit of atoms. This definition has the appeal that the cubic Si Schläfli cluster (Fig. 1a) is about $9 \AA$ in diameter, which is about the length scale at which paracrystalline medium range order in amorphous $\mathrm{Si}$ and $\mathrm{Ge}$ starts. Connected regions of identical (or patterns of) Schläfli clusters define a medium range order length scale intrinsic to the model. Marians and Hobbs [2] had earlier proposed a similar concept, which they called the local cluster, and Hobbs and co-workers have developed this idea extensively [3-5]. We prefer the term Schläfli cluster since this makes the connection with the Schläfli circuit symbol clearer.

One simple distinguishing feature of the cubic and hexagonal Schläfli clusters is their weight, or the number of vertices contained in each cluster. The cubic cluster (Fig. 1a) has a weight of 29 , whereas the hexagonal cluster (Fig. 2b) has a weight of 27. However, it emerges that this weight information is still insufficient to uniquely identify the topology of a cluster. For example, the cubic cluster in Fig. 1a could be deformed slightly so that vertices 21 and 22 are connected to create two 5-rings. The circuit symbol and weight are unaffected by this new connection. One way to detect such topological nuances is to examine all the circuits in the cluster, even those that do not involve the central vertex [3]. The cubic cluster has a total circuit count of $96 \times 6$-circuits and $12 \times 8$-circuits, whereas the hexagonal cluster has a total circuit count of $90 \times 6$-circuits and $12 \times 8$-circuits. Topological changes resulting from any deformation of the cluster will be readily detected. The above example, where vertices 21 and 22 are connected, generates the circuit count $10 \times 5$-circuits, $92 \times 6$-circuits and $10 \times 8$-circuits.

Figure 1c illustrates the differences between the Schläfli clusters generated by the Wells and O'Keeffe definitions of circuits for the faujasite framework. There are two 6-circuits passing through first nearest neighbor vertices 4 and 5 . The circuit $1,4,7,8,9,5,1$ is an open 6-circuit, whereas the circuit 1, 4, 10, 2, 11, 5, 1 is formed from two fused 4-rings $(1,4,10,2,1$ and 1,2, $11,5,1)$. The Wells definition would count both of these 6-circuits, whereas the O'Keeffe definition would count only the open 6-ring. In this instance, this particular distinction would not alter the number of atoms in the Schläfli cluster. However the 6-circuit 1, 3, 6, 4, 10,2,1 is also formed from two fused 4-rings. The Wells notation would accept this as a 6-circuit, whereas the O'Keeffe notation would reject this circuit in favor of the larger 12-ring 1,3,15, 16, 17, 18,19, $20,21,22,23,2,1$. For faujasite, the Wells circuit symbol is $4 \cdot 4 \cdot 4 \cdot 6 \cdot 6 \cdot 6_{2}$ with a weight of 14 , whereas the O'Keeffe form is $4 \cdot 4 \cdot 4 \cdot 6 \cdot 6 \cdot 12$ with a weight of 23 . In general, for 4-connected frameworks, the O'Keeffe form of the Schläfli cluster tends to provide the most local topological information [2]. However, not every nearest neighbor pair will have a fundamental circuit. In particular, 5- and 6-connected defect atoms, which can occur in molecular dynamics models of disorder, frequently have undefined fundamental circuits.

\section{CIRCUIT SYMBOL ALGORITHM}

Effective algorithms for determining the coordination sequences [11] and circuit symbols [4, 12] have already been presented in the literature. Here we outline a simpler algorithm for determining the circuit symbols. Often, amorphous models are provided as a list of atomic coordinates. The first step is to transform such structures into a bonded graph. Unless one is careful, determining bonds in large structures can be surprisingly time-consuming. Efficient algorithms for finding bonds in large structures have been published [13]. Our method for determining circuits searches the graph using a breadth-first-search strategy. We illustrate these searches in Figure 2 using a periodic, 2-dimensional, 3-connected net for simplicity. 


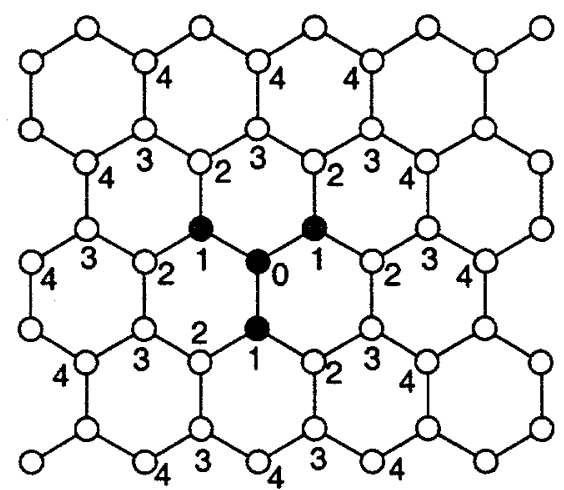

(p)

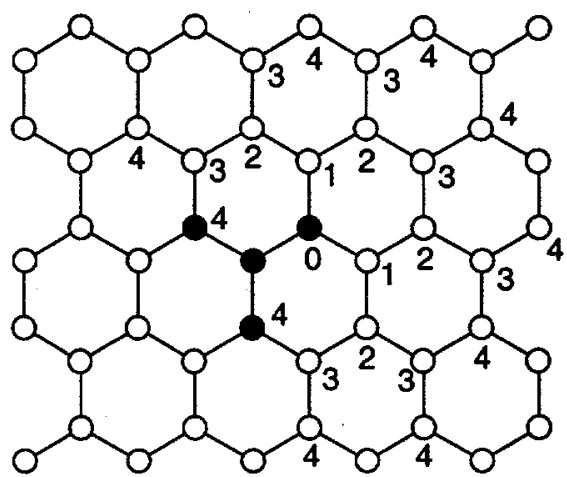

(q)

Figure 2. Diagram illustrating the algorithm for determining circuits in networks. $(p)$ Breadth-first search ( $p$ values) centered on atom of interest (black circle). (q) Breadth-first search (q values) centered on one of the first-nearest neighbor bonded atoms (gray circle 0).

We first label each atom by $p$, the number of its coordination shell around the central vertex:

1. Choose a central vertex (black circle in Figure $2 \mathrm{a}$ ). The first shell, $p=1$, is defined by the bonded neighbors (gray circles in Figure 2a).

2. For every vertex in the current shell, visit all the bonded neighbors. If the bonded vertex does not belong to the previous shell, $p-1$, or to the current shell, $p$, it must belong to the next shell. Record the new shell number $p+1$ with each vertex.

3. Repeat step 2 for the new shell $p+1$ out to a predetermined limiting radius $K$. Typically, $K=10$. The count of atoms in each shell, $N_{p}$, is the coordination sequence [11].

Next we find the Wells-type circuits:

4. Choose one of the $N, p=1$, nearest neighbor vertices (gray circles in Figure 2b). This vertex is the starting point $i$ for a second breadth-first search, which generates another set of coordination shells, $q$. The first shell, $q=1$, is defined by the bonded neighbors to this vertex $i$, but excludes the central vertex (black circle). Each vertex in the first shell is assigned a path weight of unity since there is only one path from the root vertex to each vertex in the first shell.

5. Search the first shell for occurrences of any of the other $N-1, p=1$, first-nearest neighbors. If found, this bond defines a 3-ring.

6. For every vertex in the current shell $q$, visit all the bonded neighbors. If the bonded vertex does not belong to the previous shell or to the current shell $q$, it must belong to the next shell. Record the new shell number $q+1$ with each vertex. Each vertex in shell $q+1$ inherits additively the path weights from the parent vertices in shell $q$.

7. Once complete, shell $q+1$ is searched for occurrences of any of the other $N-1, p=1$, vertices. If a $p=1$ vertex, $j$, is found, the circuit size is given by $S_{i j}=(q+1)+2$, which includes the central vertex and the two $p=1$ neighbors. The number of circuits of this size is given by the accumulated path weight at vertex $j$.

8. Repeat steps 6 and 7 until all of the other $N-1, p=1$, vertices have been found.

9. Repeat steps 4 to 8 for all $N, p=1$,vertices, until all $N(N-1) / 2$ sets of circuits have been found.

10. Count all the unique vertices that define the circuits. This is the weight of the Schläfli cluster.

11. Repeat steps 4 to 9 for each vertex in the Schläfli cluster to determine the total circuit count.

The algorithm for finding O'Keeffe-type rings is almost identical to that for Wells-type circuits given above. In step 6, there is an additional test conducted at each vertex to see if we 
have reached the aponode of any potential fundamental circuit (that is, the furthest vertex on the ring from the central vertex). Early in the breadth-first search, except for 3-rings, vertices will have the property $p=q+1$. The first vertex in any path for which $p<q+1$ is the aponode of a potential fundamental circuit. For a circuit to be a fundamental circuit, all subsequent vertices must have the property $p+q=p_{\mathrm{a}}+q_{\mathrm{a}}$ where $p_{\mathrm{a}}+q_{\mathrm{a}}$ are the values at the aponode. Vertices after the aponode that do not have this property can not possibly be on a fundamental circuit and can be excluded as parent nodes from the breadth first search. The largest fundamental circuit is limited to $2 K+1$. Not every $p=1$ nearest neighbor pair, $i j$, necessarily has a fundamental circuit, even if $K$ is arbitrarily large. Such "open" circuits are usually designated by the symbol $\infty$ [8].

Note that if only the Wells form of the Schläfli cluster is desired, the initial breadth first search for the $p$ values (Fig. 2a), steps 1-3, may be omitted. We find that in practice, it is sufficient to use the Wells-type circuit definition when computing the total circuit count of the Schläfli cluster, even if the Schläfli cluster itself was determined using O'Keeffe-type rings.

Since the circuits $C_{n}^{(i j)}$ and $C_{n}^{(j i)}$ are equivalent, the circuit-finding algorithm described above discovers each set of circuits twice. This inefficiency is hard to avoid but can be put to good use since the additional information provides a simple method for determining the number of circuits, or circuit flux, that pass through each vertex. This provides an additional degree of topological information that may be useful for discriminating ambiguous cases.

Figure 3 shows a hypothetical set of ten 14-circuits $\left(1_{10}\right)$ associated with 2 nearest neighbor vertices, which are the vertices at the top and bottom. The central vertex is the black circle on the left. The numbers beside each vertex represent the accumulated path weight at that vertex. For the clockwise circuit (Figure 3, left), the path weight is initially equal to 1 . When paths merge, the new vertex accumulates the path weights additively. In the example, the weight increases from 1 at the top, to 10 for the vertex at the bottom. Similarly, for the counter-clockwise circuit (Figure 3, middle), the path weight is equal to 1 at the bottom and accumulates to 10 at the top. The product of the path weights at each vertex that are found by traversing the circuits in both directions, gives directly the circuit flux through that vertex (see Figure 3, right).
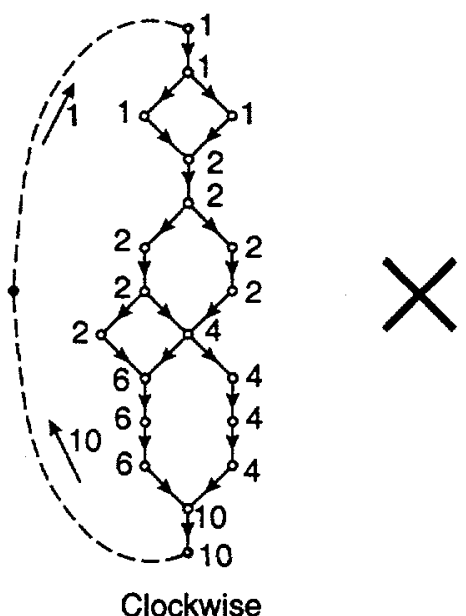

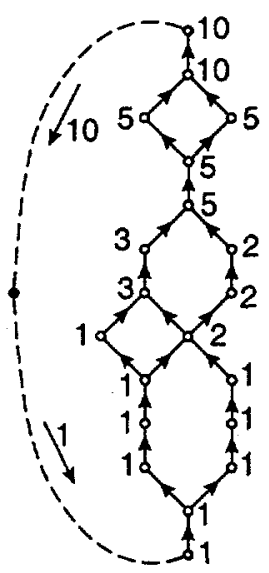

Counter-clockwise

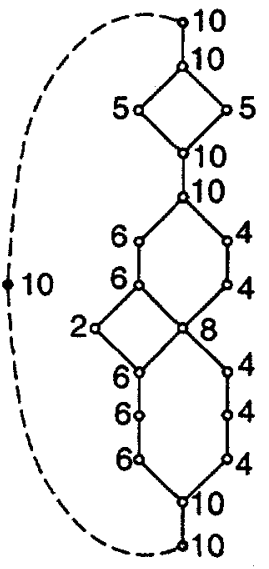

Ping flux

Figure 3. Diagram illustrating how the path weights are accrued for a set of hypothetical 14-circuits. When the accumulated path weights for the clockwise and counterclockwise versions of the same circuit are multiplied, the result is the number of circuits that "flow" through each vertex, or the circuit flux. 


\section{CONCLUSIONS}

The local cluster, or Schläfli cluster, concept of Marians and Hobbs [2] is a useful topological tool for detecting paracrystalline medium range order in models of disordered tetrahedral semiconductors [6]. Two types of Schläfli cluster can be defined depending on the definition of a circuit that is used. The Wells-type cluster uses the shortest circuits, whereas the O'Keeffe-type cluster uses the shortest rings (fundamental circuits). In general, the O'Keeffe form of the Schläfli cluster can provide more topological information, but the Wells form is simpler to implement. For cubic and hexagonal diamond-like frameworks, which are probably the most important paracrystalline topologies, the two definitions yield identical Schläfli clusters. We have presented efficient algorithms for deriving the coordination sequence, the Wells- and O'Keeffe-type circuits, and the circuit flux for a connected graph.

\section{ACKNOWLEDGMENTS}

The authors wish to thank Srivilliputhur Srinivasan for useful suggestions. P. M. V. acknowledges the support of the National Science Foundation (DMR 97-03906).

\section{REFERENCES}

1. M. M. J. Treacy, J. M. Gibson, and P. J. Keblinski, J. Non-Cryst. Sol. 231, 99-110 (1998).

2. C. S. Marians and L. W. Hobbs, J. Non-Cryst. Sol. 124, 242-253 (1990).

3. L. W. Hobbs, J. Non-Cryst. Solids 192 \& 193, 79-91 (1995).

4. L. W. Hobbs, C. E. Jesurum, V. Pulim, and B. Berger, Philos. Mag. A78, 679-711 (1998).

5. L. W. Hobbs, C. E. Jesurum, and B. Berger, The topology of silica networks, in Structure and imperfections in amorphous and crystalline $\mathrm{SiO}_{2}$, Edited by R.A.B. Devine, J.-P. Duraud, and E. Doryhée, (J. Wiley \& Sons Ltd., 2000) pp. 3-47.

6. M. M. J. Treacy, P. M. Voyles, and J. M. Gibson, J. Non-Cryst. Sol. 266, 150-155 (2000).

7. A. F. Wells, Three-Dimensional Nets and Polyhedra, (Wiley, New York, 1977).

8. M. O'Keeffe and B. G. Hyde, Crystal Structures. I. Patterns and Symmetry, (Mineralogical Society of America, Washington, 1996) Ch. 7, p 289.

9. M. M. J. Treacy, K. H. Randall, S. Rao, J. A. Perry, and D. J. Chadi, Z. Kristallogr. 212, 768791 (1997).

10. G. O. Brunner, J. Sol. State Chem. 29, 41-45 (1979).

11. R. W. Grosse-Kunstleve, G. O. Brunner, and N. J. A. Sloane, Acta Cryst. A52, 879-889 (1996).

12. K. Goetzke and H.-J. Klein, J. Non-Cryst. Sol. 127, 215-220 (1991).

13. R. W. Hockney and J. W. Eastwood, Computer simulation using particles, (McGraw-Hill, New York, 1981). 